\title{
On the acceptability of arguments and its fundamental role in nonmonotonic reasoning, logic programming and n-person games: 25 years later
}

\author{
Pietro Baroni ${ }^{\mathrm{a}}$, Francesca Toni ${ }^{\mathrm{b}}$ and Bart Verheij ${ }^{\mathrm{c}}$ \\ ${ }^{\mathrm{a}}$ University of Brescia, Italy \\ E-mail:pietro.baroni@unibs.it \\ ${ }^{\mathrm{b}}$ Imperial College London, United Kingdom \\ E-mail:f.toni@imperial.ac.uk \\ ${ }^{\mathrm{c}}$ University of Groningen, The Netherlands \\ E-mail: bart.verheij@rug.nl
}

\section{Argumentation and its fundamental role}

Arguments are pervasive and elusive at the same time.

They are pervasive as they occur continuously in many aspects of our daily life. We may find ourselves arguing with a friend about the restaurant where to go, with our doctor about which is the best therapy for a disease, or with some anonymous user of a social network about whether death penalty should be banned. Further, we often monologically argue with ourselves e.g. about the pros and cons of some important personal decision, or simply on whether to trust some news from TV.

The ability to argue appears early in a person's development: children argue with their parents without having been taught to argue (and possibly in spite of many attempts to limit this arguing attitude by the parents themselves). Consider a parent telling to a child "Stop playing, you should do your homework now". The answer "Yes, I will" is definitely not the only possible (and not the most probable) one. The child may present several objections like for instance "There is no school tomorrow" to which the parent may reply "But today is no holiday" getting in reply "But I prefer to do my homework tomorrow". Children happen also to apply more articulated argumentation strategies than just rebutting parent's claims, like appeals to a third party (e.g. grandparents) in search of a defense for their position against the parent's one.

Besides producing arguments copiously, children appear to be hungry of being fed with arguments too. The "Why?" questions they so often pose show the need of identifying justifications for claims of any 
kind, which they are not ready to accept uncritically but should be accompanied by reasons supporting them, i.e. by an argumentative structure, in order to be convincing.

The common experience with children's quarrels and questions suggests that all humans are given a mechanism for arguing, being part of the fundamental abilities one develops in the very early years of life.

It is then no surprise that argumentation pervades the history of human culture as well. It might be regarded as more than a coincidence that the most ancient poem of Western civilization, Homer's Iliad, starts with a strong argument (apparently about a relatively minor issue) among the Greek commanders besieging Troy and the poem is about its consequences on the continuation of the war.

Classic Chinese philosophers (going back to the eighth century before Christ) engaged in argumentative debates about moral issues, language and meaning, the relation between names and reality, and even about the rules of argumentation [109, Chapter 12].

The ancient philosopher Heraclitus ascribed to polemos $(\pi o \lambda \epsilon \mu o \varsigma)$, meaning conflict or war, the role of a fundamental principle underlying the nature of the universe, stating that "polemos is the father and king of everything" since all things come into being by conflict of opposites.

Besides this early radical theoretical position on the universal role of conflict, the structure and uses of arguments, in particular through the study of the disciplines of rhetoric and dialectic, have represented a constant subject of investigation since about 2500 years, ranging from the works of philosophers in classical Greece (Protagoras, Gorgias, Socrates, Plato, Aristotle just to mention some) to a flourishing of research contributions in modern and contemporary times [10,109]. The sentence Discordo ergo sum (I disagree therefore I am), giving title to the Austrian pavillion of the Biennale International Art Exhibition of Venice 2019, provides a very recent synthesis and banner of the essential role of arguing.

Pervasive and persistent as they are, arguments are elusive too.

In fact, the variety of their manifestations represents a formidable challenge for any attempt to characterize precisely what an argument is and which its boundaries are. Indeed these questions may have quite different answers in different contexts.

For instance, the Merriam-Webster dictionary for English Language learners gives three definitions of argument:

- a statement or series of statements for or against something;

- a discussion in which people express different opinions about something;

- an angry disagreement.

They capture different, but not mutually exclusive, facets of this complex phenomenon: an actual argumentation process typically fits all of them, as it involves both building some lines of reasoning leading to assert or deny some conclusions and making them interact in a dialectical context, where conflicts typically occur.

Zooming inside each item may only show further dimensions of variety. For instance, there are many ways of putting together some statements for a conclusion, as there are many forms of reasoning, like deductive, inductive, analogical, case-based and so on. Also, the statements and their relationships with the conclusion may be expressed following some canonical structure if the arguments are developed in a somehow controlled context (e.g. in a legal, technical or scientific setting) or they may be left largely implicit, leaving a significant burden of interpretation to the listener, as it often happens for arguments exchanged in daily conversations. Also, discussions where people express different opinions may follow a well-defined protocol, with rules defining turn taking and the available moves to each participant at each turn, or proceed more chaotically, as very common in real debates. 
Given the multiform nature of arguments and argumentation, the question arises on whether it is possible to develop general purpose and largely applicable theoretical and computational tools for it. A positive answer can be obtained by focusing on a few essential aspects, which are shared by all argumentation processes in spite of their many differences, while ignoring any other detail. Consider for instance the following two sets of arguments taken, introducing some necessary simplification in their formulation and of course omitting many other relevant points, from real debates available on the online platforms debatepedia.idebate.org and www.procon.org.

\section{Debate on Vegetarianism}

- Argument $A$ : A vegetarian diet should be adopted because it is generally healthier

- Argument $B$ : A vegetarian diet should not be adopted because it can promote specific health problems

- Argument $C$ : We should not eat animals because animals have rights because they are sentient beings

- Argument $D$ : Animals cannot possess rights because they have no moral judgement

\section{Debate on Vaccination}

- Argument $A$ : We should adopt vaccination programs because they can save many lives

- Argument $B$ : We should not adopt vaccination programs because vaccines can cause serious side effects as reported by independent sources

- Argument $C$ : Major medical organizations say that the claims of independent sources are unreliable because not supported by adequate scientific evidence

- Argument $D$ : Independent sources say that the claims by major medical organizations are unreliable because of conflicts of interest

Consider also a simplified example of medical debate.

\section{Debate on Therapy}

- Argument $A$ : We should give drug D1 to patient P affected by illness I since this is prescribed by the guidelines.

- Argument $B$ : We should give drug D2 to patient P affected by illness I since the outcomes of a recent clinical trial say that $\mathrm{D} 2$ is more effective than $\mathrm{D} 1$.

- Argument $C$ : The outcomes of the recent clinical trial are not based on sufficient experimental evidence according to some experts.

- Argument $D$ : The experimental evidence on which the clinical trial is based, though limited, has been considered sufficient by other experts.

While these examples concern rather different subjects it can be observed that they feature some structural similarity, the following comments being applicable to all examples.

First, each of the four items $A-D$ can be identified as an argument, i.e. as a self-standing entity providing a connection between a claim $X$ and some reasons from which it can be derived, i.e. satisfying the need of providing an answer to the question "Why $X$ ?".

Second, while each individual argument could be regarded as acceptable in isolation, it is clear that they cannot be accepted all together because, for instance, it is impossible to be vegetarian and not vegetarian at the same time or to adopt and not to adopt a vaccination program at the same time. Also 


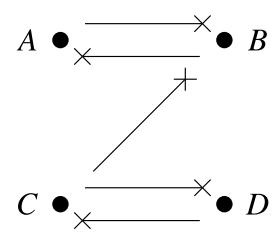

Fig. 1. Abstracting to the attacks between the four example arguments.

it would not be reasonable to accept at the same time that a given drug should be given, based on the results of some clinical trial, and that this clinical trial is not based on sufficient evidence.

It emerges rather directly that a common property in a set of arguments is that the acceptance of some of them may be incompatible with the acceptance of some other ones, which can be modeled by the existence of an attack relation between them. Structural similarities between different argumentation context can be identified focusing on this attack relation only.

For instance, in each of the above examples it is rather intuitive to state that arguments $A$ and $B$ attack each other, arguments $C$ and $D$ attack each other, and $C$ attacks $B$. These attack relations have been visualized in Fig. 1.

Thus different argumentation contexts share a fundamental question, namely identifying which arguments are acceptable and an essential feature, namely that arguments attack each other. Abstract argumentation, described in the next section, adopts the standpoint that this essential feature is a sufficient basis to define a general formalism able to provide answers to the fundamental question of acceptability of any set of arguments.

\section{Abstract argumentation}

Abstract argumentation [38,39] has been originally proposed in an effort to understand how common people, many of them may even be illiterate, carry out their reasoning. While abstract argumentation could be viewed as a formalism for capturing various approaches to commonsense reasoning (in particular non-monotonic reasoning and cooperative games), it is also a methodology for providing abstractions of problems along three dimensions: arguments, attacks and acceptability, the latter amounting to what agents have to accept given what they know (i.e. the arguments and associated attacks). Here it is interesting to note the difference between what agents accept and what they may believe. As an example, a person may not believe the decision of a supreme court, but nonetheless accept it, and a child may not believe in what parents decide but still have to accept it.

\subsection{Arguments}

Naturally, the first task of any practical argument exchange is to construct arguments. Humans learn to form arguments since early childhood.

Mathematical proofs, perhaps the most extensively studied form of arguments, teach us that arguments start with basic facts and axioms and then develop further by using rules, lemmas, theorems, etc. The impression of mathematical arguments on us is so strong that, in the early days of AI, it seemed that the prevailing belief was that AI reasoning systems should be just forms of mathematical arguments. Toulmin [107] recognized early that practical reasoning is not based on mathematical arguments only. AI researchers also recognized in the seventies and eighties that we need more than mathematical arguments 
to build practical reasoning systems, in particular in logic programming and non-monotonic logic (see e.g. [54]).

The structure of arguments may be complex in general, and different argumentation models choose different structures for arguments. For example, in Pollock's work (with [82] an early paper, further developed in $[83,84]$ ), the focus is on prima facie reasons and associated rebutting and undercutting defeaters. Simari and Loui [100] combine structured arguments with specificity (see also [69]). Vreeswijk [116] studies structured arguments and their conclusive force. In ASPIC + [79,85] arguments are built from rules of two types (strict and defeasible) and premises of various kinds (e.g. ordinary premises and axioms). Deductive argumentation [18] uses arguments consisting of premises and a conclusion with a focus on consistency management. In assumption-based argumentation [21] arguments amount to deductions with inference rules from assumptions (i.e. singled-out "arguable" sentences in the underlying language), presented in a forward, backward or tree-like format, interchangeably [106].

For instance, in the earlier debate on therapy, there was an argument for giving drug D1 to patient $\mathrm{P}$ based on the guidelines, and another argument for giving drug D2 based on a recent clinical trial. Different models use different representations of the structure of those arguments, using reasons, rules, assumptions, logical deductions in various combinations.

\subsection{Attacks}

The notion of attack is core in abstract argumentation, as without disagreement there is no arguing. In the examples, we already discussed how arguments A, B, C and D are attacking each other (shown graphically in Fig. 1).

Attacks may originate from several sources and indeed several types of attack have been identified in the literature. In particular, often rebuttal and undercutting attacks are distinguished (following [82]): the rebutting type unearths disagreement between arguments with conflicting conclusions, whereas the undercutting type emphasises an attack on the connection between reason and conclusion. For example, in the earlier debate on therapy, Arguments A and B can be interpreted as rebutting one another, one supporting giving drug D1, the other giving D2. Argument C can be interpreted as undercutting Argument $\mathrm{B}$, raising doubt about the reason why drug D2 would be more effective.

Different argumentation models use alternative ways to include kinds of attack, sometimes distinguishing more kinds, sometimes reducing different kinds to a single formal structure.

\subsection{Argument acceptance}

A cornerstone of abstract argumentation is that argument acceptance can be evaluated taking into account the attack relation only, independently of any other underlying detail, thus ensuring a uniform applicability to a wide variety of situations. Intuitively, arguments are accepted if they can be defended against attacks. For instance, if an argument $\mathrm{P}$ is attacked by an argument $\mathrm{Q}$, the first argument can be defended against the attack by a third argument $\mathrm{R}$ that attacks the second argument $\mathrm{Q}$. By itself, the argument $\mathrm{P}$ would be successfully attacked or defeated by $\mathrm{Q}$, but, considering also the counterattack by $\mathrm{R}$, the argument $\mathrm{Q}$ is no longer defeated and said to be reinstated. As a pair, the arguments $\mathrm{P}$ and $\mathrm{R}$ can withstand the attack by $\mathrm{Q}$.

In abstract argumentation, this idea of a collection of arguments that can defend themselves against attack is captured in the central notion of an admissible set. An admissible set of arguments is a set that contains no arguments that attack themselves or other arguments in the set and that contains a 
counterattack against all attack of arguments in the set. For instance, the set consisting of the arguments $\mathrm{P}$ and $\mathrm{R}$ is admissible since $\mathrm{P}$ and $\mathrm{R}$ do not attack each other (or themselves) and $\mathrm{R}$ defends $\mathrm{P}$ against the attack by $\mathrm{Q}$. The set consisting of only $\mathrm{P}$ is not admissible since there is no defence against $\mathrm{Q}$, and the set consisting of $\mathrm{P}$ and $\mathrm{R}$ is not since $\mathrm{R}$ attacks $\mathrm{P}$.

In abstract argumentation, several special kinds of admissible set are distinguished, referred to as extensions, expressing different perspectives on the arguments that are collectively acceptable. Here we discuss the stable, the preferred and grounded extensions:

- Stable extensions. A stable extension is a set of arguments that contains no arguments that attack each other and that also attacks every argument that is not in the set. Given a stable extension, one could say that all conflicts between arguments are resolved: either an argument is in the collectively defensible set, or it is attacked by the set. Stable extensions do not always exist, and sometimes there are many of them, suggesting alternative ways of resolving conflicts. For instance, the attacks between the example arguments A, B, C and D lead to three alternative stable extensions, one in which $\mathrm{A}$ and $\mathrm{C}$ are accepted, one containing $\mathrm{A}$ and $\mathrm{D}$, and the third one $\mathrm{B}$ and $\mathrm{D}$.

- Preferred extensions. A preferred extension is a set that is a maximal admissible set, in the sense that after adding any additional argument the set is no longer admissible. There always exists at least one preferred extension - even when there is no stable extension, suggesting a way to solve as many conflicts as possible. Like for stable extensions, there can be several alternative preferred extensions. In the example, there are three preferred extensions, coinciding with the stable extensions.

- Grounded extensions. The grounded extension - there is always exactly one - expresses a set of acceptable arguments about which there is no ambiguity. The grounded extension can be constructed by accepting all arguments that are not attacked at all, then adding all arguments that are defended by the already accepted arguments, and so on. In the example, the grounded extension is empty, since all arguments are attacked by at least one other argument.

In Dung's original work, these three kinds of argument acceptance were defined, and also complete extensions. In follow-up work, further options were explored [12,26,41,44,112] (see [8] for an overview).

\section{Beyond abstract argumentation}

Abstract argumentation became the basis of extensive research on the formal and computational modeling of argumentation. Google Scholar shows more than 4000 citations to [39]. In the following, we discuss further argumentative notions, argumentation software and algorithms, argumentation schemes and dialogue, and further applications.

\subsection{Further argumentative notions}

Abstract argumentation provided the tools to show how several formal reasoning concepts could be interpreted in terms of attacks between arguments. In particular, in [39], it is shown how Reiter's default logic [95], Pollock's defeasible reasoning [82] and logic programming [55] can be understood as instances of abstract argumentation.

In later work, abstract argumentation was also used as a basic level of expressiveness focusing only on attacks between arguments. The expressiveness was then extended to incorporate other argumentative notions. For instance, support was added, and nesting of support and attack [9,28,36,77,113]. We already mentioned different approaches to argument structure (Section 2.1, and see [17]), some directly 
building on [39], others indirectly. Further notions that have been studied include preference between arguments $[2,3,37,77]$, weights and probabilities $[42,45,53,62,63,72,103]$, dialogues $[40,50,74]$ (with historic roots in [13]), practical reasoning [6,14,43,73], the role of the audience [15] (connecting to [81]), belief revision [49,51], negotiation [80,88], argumentation schemes [19,114] (building on ideas in argumentation theory [57,117,119]), argument diagrams [94,115] (following in the footsteps of [107]), burden of proof [59], logic programming [56,67] and generalized views on the relations between arguments $[22,23,27]$. By the wealth of formalisms, properties of argumentation have been studied in order to systematize and for a deepened understanding $[1,7,11,108]$.

\subsection{Argumentation software and algorithms}

Already before abstract argumentation, software was developed that performed argumentative reasoning. A prominent example is Pollock's work on OSCAR [83,84]. Inspired by his research in the philosophy of knowledge, he noticed that AI's nonmonotonic logic coincided with philosophy's defeasible reasoning (see also [70]), and he started building an intelligent computational agent, called OSCAR, able to think in terms of defeasible arguments.

Another kind of argumentation software focused on argument diagrams, often using boxes for the statements made in an argument and arrows for the relations between the statements. Reed and Rowe's Araucaria [91] has been influential and allows for the diagrammatic analysis of argumentative texts. The Zeno system by Gordon and Karacapilidis [58] allowed for argument analysis in terms of issues, questions of fact, positions and arguments, as in the influential IBIS approach to so-called wicked problems with little structure and an open-ended solution space [98]. Another argument diagramming tool is Van Gelder's Rationale tool [111], which is so user-friendly that it could be successfully applied in critical thinking education. Verheij's ArguMed software combines argument diagramming with evaluation in terms of stable semantics [115]. Dispute derivations of various kinds have also been defined, e.g. as in [35,105], to provide constructive ways to build argumentative debates about queries.

As a common backbone of argumentation software, supporting open source sharing and machine readability, representation formats were developed, in particular the argument markup language AML, the argument interchange format AIF [32] and the argument description framework ArgDF [90].

The formal properties of computational problems in argumentation, the study of their complexity, and the development of efficient algorithms to solve them have also received considerable attention in the field. In particular, computational complexity of standard computational problems with reference to different argumentation semantics has been systematically analyzed [46,47], abstract argumentation has been encoded in answer-set programming [48] and methods for solving argumentation problems have been studied [29,30]. Recently a series of competitions have stimulated the development of efficient algorithms for solving problems defined in terms of abstract argumentation semantics (see [71,104]).

\subsection{Argumentation schemes and dialogue}

Abstract argumentation inspired research into the formalization of argumentation schemes as they were developed in non-formal argumentation theory and critical thinking research [57,117]. Argumentation schemes (also referred to as argument schemes) can be regarded as a conceptual generalization of the rules of inference of formal logic, such as Modus ponens. While rules of inference are formal and logically valid, argumentation schemes are often concrete and allow for exceptions. Argumentation schemes typically come with premises, a conclusion and critical questions. For example, an argumentation scheme for witness testimony could come with the premise 'The witness says that P', the conclusion 
'The statement $\mathrm{P}$ is true' and critical question 'Isn't the witness mistaken about P?'. Several proposals for the formal and computational modeling of argumentation schemes exist and Walton, Reed and Macagno have collected an extensive catalogue of argumentation schemes [119].

The critical questions of argumentation schemes point to the dialogue setting in which argumentation often takes place $[13,107,110]$. When a proponent makes the claim 'The suspect committed the crime', an opponent can ask why that claim is made, and when the proponent then offers support for the claim 'The witness saw that the suspect committed the crime', the opponent can continue with asking the critical question 'Isn't the witness mistaken about P?'.

Walton and Krabbe [118] proposed a typology of argumentation dialogues, such as deliberation, negotiation and persuasion, with different starting points and goals, several of which have been studied formally. Semantics of abstract argumentation have been characterized in terms of winning strategies of dialogue protocols [78].

\subsection{Further applications}

By the prominent role of argumentation in the law, where parties defend their own positions and challenge those of their opponents, there has been much interaction between research into the formal and computational modeling of argumentation and of legal reasoning [97].

Three types of legal reasoning have been studied in terms of argumentation, each with its specific characteristics: rule-based, case-based and evidence-based reasoning. In rule-based reasoning in the law, arguments are built by applying rules, and can be attacked by supporting exceptions to the rules [86]. In case-based reasoning in the law, precedent cases are used to discover possible arguments in new cases, that can then be decided in analogy with the precedent case by showing factors shared between precedent and current case $[4,96]$. Attacks of such analogy-based arguments can be found by showing distinguishing factors between precedent and current case. In evidence-based reasoning, arguments are used to interpret and evaluate conflicting, incomplete and uncertain evidence [20]. Research into argument-based legal reasoning have inspired connections between arguments, rules and cases and between arguments, scenarios and probabilities.

The formal and computational advances inspired by abstract argumentation have been applied in a variety of settings. We already mentioned argumentation software and its use in critical thinking education. Also models of legal argumentation have been applied to real world cases. Argumentation has been applied as the foundation of computational tools for medical diagnosis [52], for health promotion [60], for evidence aggregation [65] and for the design of software or other artefacts [24]. Recently the mining of argumentative information from natural language texts is being studied [25,34,68,75,76,99,101]. Other application domains are persuasion technology [61,64], ontology engineering [102] and discussion mediation [66]. Finally, several combinations of argumentation and machine learning have been studied (e.g. see [33]).

\section{Conclusion}

The study of argumentation, in its historical, formal and applied development shows its fundamental role in applications in a diversity of domains and explains how people from different cultures, ages and times can understand each other as long as they are willing to peacefully resolve conflicts by focusing on 
arguments and how they attack each other. Without any claim to completeness, we have shown examples of the breadth and depth of research into computational argumentation in this foreword. ${ }^{1}$

The work on abstract argumentation [38,39] has inspired many researchers in their formal, computational and applied efforts towards achieving a better understanding of the fascinating topic of argumentation in all its complexity and nuances. This was also recognized by the recent Artificial Intelligence Journal Classic Paper Award (announced at IJCAI 2018) for the pioneering paper published in 1995.

Celebrating 25 years of fruitful research having roots in and taking inspiration from this work, this special issue provides a representative collection of contributions from the field.

The first two papers exemplify the richness of possible connections between abstract argumentation and other formalisms.

In particular, the paper Abstract Argumentation and (Optimal) Stable Marriage Problems, by Stefano Bistarelli and Francesco Santini, provides developments about the connection between several forms of abstract argumentation and several kinds of stable matching problems, thus extending the basic results on this topic presented in [39] and confirming the fertility of the modeling approach adopted there.

Then, the paper Logical Theories and Abstract Argumentation: A Survey of Existing Works, by Philippe Besnard, Claudette Cayrol, and Marie-Christine Lagasquie-Schiex, deals with connections between abstract argumentation and logic and provides a comprehensive survey of works where logic has been used for capturing various aspects or parts of Dung's argumentation.

The following three papers provide technical contributions concerning extensions and variants sprouting from the original argumentation frameworks of [39].

First, the paper Similarity Notions in Bipolar Abstract Argumentation by Paola Daniela Budán, Melisa Gisselle Escañuela Gonzalez, Maximiliano Celmo David Budán, Maria Vanina Martinez and Guillermo Ricardo Simari, investigates similarity measures for arguments and their use in the context of frameworks encompassing both relations of attack and support.

Two contributions then deal with the generalization of argumentation frameworks called Abstract Dialectical Frameworks (ADFs).

The paper Structural Constraints for Dynamic Operators in Abstract Argumentation by Johannes Wallner aims to support the development of dynamic operators on formal argumentation models by studying constraints to be imposed on the modification of the structure of ADFs in order to incorporate information that has been abstracted away in the modeling but should be taken into account in a dynamic context.

The paper Investigating Subclasses of Abstract Dialectical Frameworks by Martin Diller, Atefeh Keshavarzi Zafarghandi, Thomas Linsbichler, and Stefan Woltran, explores the properties of families of ADFs obeying specific constraints, like acyclicity and symmetry, and draws a comparison with the properties induced by the same kind of constraints in Dung's argumentation frameworks.

The special issue is closed by the paper Before and After Dung: Argumentation in AI and Law, by Trevor Bench-Capon, providing a perspective on the unifying role of abstract argumentation in the key application field of AI and Law and discussing how the demands of legal systems have stimulated developments to the original proposal.

Altogether we believe these contributions are witnesses both of the role played by Dung's abstract argumentation since its appearance to the present and of the wealth of further developments to be expected in the future.

\footnotetext{
${ }^{1}$ More can be found in reviews and handbooks, such as $[5,10,16,31,87,89,92,93,109]$.
} 
In conclusion, as editors we would like to express our deepest gratitude to all the authors and reviewers whose efforts have made this special issue possible and to Phan Minh Dung for having provided insightful suggestions and comments on this foreword and for his outstanding and continuing contribution to the field.

\section{References}

[1] L. Amgoud and M. Caminada, On the evaluation of argumentation formalisms, Artificial Intelligence 172 (2007), 286310. doi:10.1016/j.artint.2007.02.003.

[2] L. Amgoud and C. Cayrol, A reasoning model based on the production of acceptable arguments, Annals of Mathematics and Artificial Intelligence 34(1-3) (2002), 197-215. doi:10.1023/A:1014490210693.

[3] L. Amgoud and S. Vesic, Rich preference-based argumentation frameworks, International Journal of Approximate Reasoning 55(2) (2014), 585-606. doi:10.1016/j.ijar.2013.10.010.

[4] K.D. Ashley, Modeling Legal Arguments: Reasoning with Cases and Hypotheticals, The MIT Press, Cambridge (Massachusetts), 1990.

[5] K. Atkinson, P. Baroni, M. Giacomin, A. Hunter, H. Prakken, C. Reed, G. Simari, M. Thimm and S. Villata, Toward artificial argumentation, AI Magazine 38(3) (2017), 25-36. doi:10.1609/aimag.v38i3.2704.

[6] K. Atkinson and T.J.M. Bench-Capon, Practical reasoning as presumptive argumentation using action based alternating transition systems, Artificial Intelligence 171 (2007), 855-874. doi:10.1016/j.artint.2007.04.009.

[7] P. Baroni, G. Boella, F. Cerutti, M. Giacomin, L. van der Torre and S. Villata, On the input/output behavior of argumentation frameworks, Artificial Intelligence 217 (2014), 144-197. doi:10.1016/j.artint.2014.08.004.

[8] P. Baroni, M. Caminada and M. Giacomin, An introduction to argumentation semantics, The Knowledge Engineering Review 26(4) (2011), 365-410. doi:10.1017/S0269888911000166.

[9] P. Baroni, F. Cerutti, M. Giacomin and G. Guida, AFRA: Argumentation framework with recursive attacks, International Journal of Approximate Reasoning 52(1) (2011), 19-37. doi:10.1016/j.ijar.2010.05.004.

[10] P. Baroni, D. Gabbay, M. Giacomin and L. van der Torre (eds), Handbook of Formal Argumentation, College Publications, London, 2018.

[11] P. Baroni and M. Giacomin, On principle-based evaluation of extension-based argumentation semantics, Artificial Intelligence 171(10-15) (2007), 675-700. doi:10.1016/j.artint.2007.04.004.

[12] P. Baroni, M. Giacomin and G. Guida, SCC-recursiveness: A general schema for argumentation semantics, Artificial Intelligence 168(1) (2005), 162-210. doi:10.1016/j.artint.2005.05.006.

[13] E.M. Barth and E.C.W. Krabbe, From Axiom to Dialogue. A Philosophical Study of Logics and Argumentation, De Gruyter, New York (New York), 1982.

[14] T.J.M. Bench-Capon, Persuasion in practical argument using value-based argumentation frameworks, Journal of Logic and Computation 13(3) (2003), 429-448. doi:10.1093/logcom/13.3.429.

[15] T.J.M. Bench-Capon, S. Doutre and P.E. Dunne, Audiences in argumentation frameworks, Artificial Intelligence 171(1) (2007), 42-71. doi:10.1016/j.artint.2006.10.013.

[16] T.J.M. Bench-Capon and P.E. Dunne, Argumentation in artificial intelligence, Artificial Intelligence 171(10-15) (2007), 619-641. doi:10.1016/j.artint.2007.05.001.

[17] P. Besnard, A.J. García, A. Hunter, S. Modgil, H. Prakken, G.R. Simari and F. Toni, Introduction to structured argumentation, Argument and Computation 5 (2014), 1-4. doi:10.1080/19462166.2013.869764.

[18] P. Besnard and A. Hunter, Elements of Argumentation, The MIT Press, Cambridge (Massachusetts), 2008.

[19] F.J. Bex, H. Prakken, C.A. Reed and D.N. Walton, Towards a formal account of reasoning about evidence: Argumentation schemes and generalisations, Artificial Intelligence and Law 11(2/3) (2003), 125-165. doi:10.1023/B:ARTI. 0000046007.11806.9a.

[20] F.J. Bex, P.J. van Koppen, H. Prakken and B. Verheij, A hybrid formal theory of arguments, stories and criminal evidence, Artificial Intelligence and Law 18 (2010), 1-30. doi:10.1007/s10506-010-9092-x.

[21] A. Bondarenko, P.M. Dung, R.A. Kowalski and F. Toni, An abstract, argumentation-theoretic approach to default reasoning, Artificial Intelligence 93 (1997), 63-101. doi:10.1016/S0004-3702(97)00015-5.

[22] G. Brewka, H. Strass, S. Ellmauthaler, J.P. Wallner and S. Woltran, Abstract dialectical frameworks revisited, in: Proc. of the 23rd International Joint Conference on Artificial Intelligence (IJCAI 2013), 2013, pp. 803-809.

[23] G. Brewka and S. Woltran, Abstract dialectical frameworks, in: Proc. of the 12th International Conference on the Principles of Knowledge Representation and Reasoning (KR 2010), 2010, pp. 102-111.

[24] S. Buckingham Shum and N. Hammond, Argumentation-based design rationale. What use at what cost?, International Journal of Human-Computer Studies 40(4) (2000), 603-652. 
[25] E. Cabrio and S. Villata, Five years of argument mining: A data-driven analysis, in: Proc. of the 27th International Joint Conference on Artificial Intelligence (IJCAI 2018), J. Lang, ed., 2018, pp. 5427-5433.

[26] M. Caminada, Semi-stable semantics, in: Proc. of the 1st International Conference on Computational Models of Argument (COMMA 2006), P.E. Dunne and T.J.M. Bench-Capon, eds, IOS Press, Amsterdam, 2006, pp. 121-130.

[27] M. Caminada and D.M. Gabbay, A logical account of formal argumentation, Studia Logica 93(2-3) (2009), 109. doi:10. 1007/s11225-009-9218-x.

[28] C. Cayrol and M.C. Lagasquie-Schiex, Bipolar abstract argumentation systems, in: Argumentation in Artificial Intelligence, I. Rahwan and G.R. Simari, eds, Springer, Berlin, 2009, pp. 65-84. doi:10.1007/978-0-387-98197-0_4.

[29] F. Cerutti, S.A. Gaggl, M. Thimm and J.P. Wallner, Foundations of implementations for formal argumentation, in: Handbook of Formal Argumentation, P. Baroni, D. Gabbay, M. Giacomin and L. van der Torre, eds, College Publications, London, 2018, pp. 689-767.

[30] G. Charwat, W. Dvořák, S.A. Gaggl, J.P. Wallner and S. Woltran, Methods for solving reasoning problems in abstract argumentation - a survey, Artificial Intelligence 220 (2015), 28-63. doi:10.1016/j.artint.2014.11.008.

[31] C.I. Chesñevar, A.G. Maguitman and R.P. Loui, Logical models of argument, ACM Computing Surveys 32(4) (2000), 337-383. doi:10.1145/371578.371581.

[32] C.I. Chesñevar, J. McGinnis, S. Modgil, I. Rahwan, C. Reed, G. Simari, M. South, G.A.W. Vreeswijk and S. Willmott, Towards an argument interchange format, The Knowledge Engineering Review 21(4) (2006), 293-316. doi:10.1017/ S0269888906001044.

[33] O. Cocarascu and F. Toni, Argumentation for machine learning: A survey, in: Proc. of the 6th International Conference on Computational Models of Argument (COMMA 2016), P. Baroni, T.F. Gordon, T. Scheffler and M. Stede, eds, IOS Press, Amsterdam, 2016, pp. 219-230.

[34] O. Cocarascu and F. Toni, Combining deep learning and argumentative reasoning for the analysis of social media textual content using small data sets, Computational Linguistics 44(4) (2018). doi:10.1162/coli_a_00338.

[35] R. Craven and F. Toni, Argument graphs and assumption-based argumentation, Artificial Intelligence 233 (2016), 1-59. doi:10.1016/j.artint.2015.12.004.

[36] K. Cyras, C. Schulz and F. Toni, Capturing bipolar argumentation in non-flat assumption-based argumentation, in: Proc. of the 20th International Conference on Principles and Practice of Multi-Agent Systems (PRIMA 2017), B. An, A.L.C. Bazzan, J. Leite, S. Villata and L. van der Torre, eds, Lecture Notes in Computer Science, Vol. 10621, Springer, 2017, pp. 386-402. doi:10.1007/978-3-319-69131-2_23.

[37] K. Cyras and F. Toni, ABA+: Assumption-based argumentation with preferences, in: Proc. of the 15th International Conference on Principles of Knowledge Representation and Reasoning (KR 2016), B. Baral, J.P. Delgrande and F. Wolter, eds, AAAI Press, 2016, pp. 553-556.

[38] P.M. Dung, On the acceptability of arguments and its fundamental role in nonmonotonic reasoning and logic programming, in: Proc. of the 13th International Joint Conference on Artificial Intelligence (IJCAI 93), 1993, pp. 852-859.

[39] P.M. Dung, On the acceptability of arguments and its fundamental role in nonmonotonic reasoning, logic programming and n-person games, Artificial Intelligence 77 (1995), 321-357. doi:10.1016/0004-3702(94)00041-X.

[40] P.M. Dung, R.A. Kowalski and F. Toni, Dialectic proof procedures for assumption-based, admissible argumentation, Artificial Intelligence 170(2) (2006), 114-159. doi:10.1016/j.artint.2005.07.002.

[41] P.M. Dung, P. Mancarella and F. Toni, Computing ideal sceptical argumentation, Artificial Intelligence 171(10) (2007), 642-674. doi:10.1016/j.artint.2007.05.003.

[42] P.M. Dung and P.M. Thang, Towards (probabilistic) argumentation for jury-based dispute resolution, in: Proc. of the 3rd International Conference on Computational Models of Argument (COMMA 2010), P. Baroni, F. Cerutti, M. Giacomin and G.R. Simari, eds, IOS Press, Amsterdam, 2010, pp. 171-182.

[43] P.M. Dung, F. Toni and P. Mancarella, Some design guidelines for practical argumentation systems, in: Proc. of the 3rd International Conference on Computational Models of Argument (COMMA 2010), P. Baroni, F. Cerutti, M. Giacomin and G.R. Simari, eds, IOS Press, Amsterdam, 2010, pp. 183-194.

[44] P.E. Dunne and T.J.M. Bench-Capon, Coherence in finite argument systems, Artificial Intelligence 141(1-2) (2002), 187-203. doi:10.1016/S0004-3702(02)00261-8.

[45] P.E. Dunne, A. Hunter, P. McBurney, S. Parsons and M. Wooldridge, Weighted argument systems: Basic definitions, algorithms, and complexity results, Artificial Intelligence 175(2) (2011), 457-486. doi:10.1016/j.artint.2010.09.005.

[46] P.E. Dunne and M. Wooldridge, Complexity of abstract argumentation, in: Argumentation in Artificial Intelligence, I. Rahwan and G.R. Simari, eds, Springer, Berlin, 2009, pp. 85-104. doi:10.1007/978-0-387-98197-0_5.

[47] W. Dvorák and P.E. Dunne, Computational problems in formal argumentation and their complexity, in: Handbook of Formal Argumentation, P. Baroni, D. Gabbay, M. Giacomin and L. van der Torre, eds, College Publications, London, 2018, pp. 631-687.

[48] U. Egly, S. Gaggl and S. Woltran, Answer-set programming encodings for argumentation frameworks, Argument and Computation 1(2) (2010), 147-177. doi:10.1080/19462166.2010.486479. 
[49] M.A. Falappa, G. Kern-Isberner and G.R. Simari, Explanations, belief revision and defeasible reasoning, Artificial Intelligence 141(1-2) (2002), 1-28. doi:10.1016/S0004-3702(02)00258-8.

[50] X. Fan and T. Toni, A general framework for sound assumption-based argumentation dialogues, Artificial Intelligence 216 (2014), 20-54. doi:10.1016/j.artint.2014.06.001.

[51] E. Fermé, D.M. Gabbay and G.R. Simari (eds), Trends in Belief Revision and Argumentation Dynamics, College Publications, London, 2013.

[52] J. Fox and S. Das, Safe and Sound: Artificial Intelligence in Hazardous Applications, The MIT Press, Cambridge (Massachusetts), 2000.

[53] D.M. Gabbay, Equational approach to argumentation networks, Argument \& Computation 3(2-3) (2012), 87-142. doi:10.1080/19462166.2012.704398.

[54] D.M. Gabbay, C.J. Hogger and J.A. Robinson (eds), Handbook of Logic in Artificial Intelligence and Logic Programming. Volume 3. Nonmonotonic Reasoning and Uncertain Reasoning, Clarendon Press, Oxford, 1994.

[55] D.M. Gabbay, C.J. Hogger and J.A. Robinson (eds), Logic Programming, Handbook of Logic in Artificial Intelligence and Logic Programming., Vol. 5, Clarendon Press, Oxford, 1997.

[56] A.J. García and G.R. Simari, Defeasible logic programming: An argumentative approach, Theory and Practice of Logic Programming 4(2) (2004), 95-138. doi:10.1017/S1471068403001674.

[57] B.J. Garssen, Argument schemes, in: Crucial Concepts in Argumentation Theory, F.H. Van Eemeren, ed., Amsterdam University Press, Amsterdam, 1997, pp. 81-99.

[58] T.F. Gordon and N. Karacapilidis, The zeno argumentation framework, in: Proc. of the 6th International Conference on Artificial Intelligence and Law (ICAIL 1997), ACM Press, New York (New York), 1997, pp. 10-18.

[59] T.F. Gordon, H. Prakken and D.N. Walton, The Carneades model of argument and burden of proof, Artificial Intelligence 171(10-15) (2007), 875-896. doi:10.1016/j.artint.2007.04.010.

[60] F. Grasso, A. Cawsey and R. Jones, Dialectical argumentation to solve conflicts in advice giving. A case study in the promotion of healthy nutrition, International Journal of Human-Computer Studies 53(6) (2000), 1077-1115. doi:10. 1006/ijhc.2000.0429.

[61] E. Hadoux and A. Hunter, Comfort or safety? Gathering and using the concerns of a participant for better persuasion, Argument \& Computation 10(2) (2019), 113-147. doi:10.3233/AAC-191007.

[62] A. Hunter, A probabilistic approach to modelling uncertain logical arguments, International Journal of Approximate Reasoning 54 (2012), 47-81. doi:10.1016/j.ijar.2012.08.003.

[63] A. Hunter, Probabilistic qualification of attack in abstract argumentation, International Journal of Approximate Reasoning 55 (2014), 607-638. doi:10.1016/j.ijar.2013.09.002.

[64] A. Hunter, Towards a framework for computational persuasion with applications in behaviour change, Argument \& Computation 9(1) (2018), 15-40. doi:10.3233/AAC-170032.

[65] A. Hunter and M. Williams, Qualitative evidence aggregation using argumentation, in: Proc. of the 3rd International Conference on Computational Models of Argument (COMMA 2010), P. Baroni, F. Cerutti, M. Giacomin and G.R. Simari, eds, IOS Press, Amsterdam, 2010, pp. 287-298.

[66] M. Janier, M. Snaith, K. Budzynska, J. Lawrence and C. Reed, A system for dispute mediation: The mediation dialogue game, in: Proc. of the 6th International Conference on Computational Models of Argument (COMMA 2016), P. Baroni, T.F. Gordon, T. Scheffler and M. Stede, eds, IOS Press, Amsterdam, 2016, pp. 351-358.

[67] K.C. Kakas and F. Toni, Computing argumentation in logic programming, Journal of Logic and Computation 9(4) (1999), 515-562. doi:10.1093/logcom/9.4.515.

[68] M. Lippi and P. Torroni, Context-independent claim detection for argument mining, in: Proc. of the 24th International Joint Conference on Artificial Intelligence (IJCAI 2015), Q. Yang and M. Wooldridge, eds, 2015, pp. 185-191.

[69] R.P. Loui, Defeat among arguments: A system of defeasible inference, Computational Intelligence 3(1) (1987), 100-106. doi:10.1111/j.1467-8640.1987.tb00178.x.

[70] R.P. Loui, Hart's critics on defeasible concepts and ascriptivism, in: Proc. of the 5th International Conference on Artificial Intelligence and Law (ICAIL 1995), ACM Press, New York (New York), 1995, pp. 21-30.

[71] J.G. Mailly and M. Maratea, Assessment of benchmarks for abstract argumentation, Argument and Computation 10(2) (2019), 107-112. doi:10.3233/AAC-192101.

[72] P.A. Matt and F. Toni, A game-theoretic measure of argument strength for abstract argumentation, in: Proc. of the 11th European Conference on Logics in Artificial Intelligence (JELIA 2008), S. Hölldobler, C. Lutz and H. Wansing, eds, Lecture Notes in Computer Science, Vol. 5293, Springer, 2008, pp. 285-297. doi:10.1007/978-3-540-87803-2_24.

[73] P. McBurney, D. Hitchcock and S. Parsons, The eightfold way of deliberation dialogue, International Journal of Intelligent Systems 22(1) (2007), 95-132. doi:10.1002/int.20191.

[74] P. McBurney and S. Parsons, Games that agents play: A formal framework for dialogues between autonomous agents, Journal of Logic, Language and Information 11(3) (2002), 315-334. doi:10.1023/A:1015586128739. 
[75] R. Mochales Palau and M.F. Moens, Argumentation mining: The detection, classification and structure of arguments in text, in: Proc. of the 12th International Conference on Artificial Intelligence and Law (ICAIL 2009), ACM Press, New York (New York), 2009, pp. 98-107.

[76] R. Mochales Palau and M.F. Moens, Argumentation mining, Artificial Intelligence and Law 19(1) (2011), 1-22. doi:10. 1007/s10506-010-9104-x.

[77] S. Modgil, Reasoning about preferences in argumentation frameworks, Artificial Intelligence 173(9) (2009), 901-934. doi:10.1016/j.artint.2009.02.001.

[78] S. Modgil and M. Caminada, Proof theories and algorithms for abstract argumentation frameworks, in: Argumentation in Artificial Intelligence, I. Rahwan and G.R. Simari, eds, Springer, 2009, pp. 105-129. doi:10.1007/978-0-387-981970_6.

[79] S. Modgil and H. Prakken, A general account of argumentation with preferences, Artificial Intelligence 195 (2013), 361-397. doi:10.1016/j.artint.2012.10.008.

[80] S. Parsons, C. Sierra and N. Jennings, Agents that reason and negotiate by arguing, Journal of Logic and Computation 8(3) (1998), 261-292. doi:10.1093/logcom/8.3.261.

[81] C. Perelman and L. Olbrechts-Tyteca, The New Rhetoric: A Treatise on Argumentation [English Translation of la Nouvelle Rhétorique: Traité de L'argumentation], University of Notre Dame Press, Notre Dame (Indiana), 1958/1969.

[82] J.L. Pollock, Defeasible reasoning, Cognitive Science 11(4) (1987), 481-518. doi:10.1207/s15516709cog1104_4.

[83] J.L. Pollock, Cognitive Carpentry: A Blueprint for How to Build a Person, The MIT Press, Cambridge (Massachusetts), 1995.

[84] J.L. Pollock, Defeasible reasoning and degrees of justification, Argument and Computation 1(1) (2010), 7-22. doi:10. 1080/19462161003728921.

[85] H. Prakken, An abstract framework for argumentation with structured arguments, Argument and Computation 1(2) (2010), 93-124. doi:10.1080/19462160903564592.

[86] H. Prakken and G. Sartor, A dialectical model of assessing conflicting arguments in legal reasoning, Artificial Intelligence and Law 4 (1996), 331-368. doi:10.1007/BF00118496.

[87] H. Prakken and G.A.W. Vreeswijk, Logics for defeasible argumentation, in: Handbook of Philosophical Logic, D.M. Gabbay and F. Guenthner, eds, Vol. 4, 2nd edn, Kluwer Academic Publishers, Dordrecht, 2002, pp. 218-319.

[88] I. Rahwan, S.D. Ramchurn, N.R. Jennings, P. McBurney, S. Parsons and L. Sonenberg, Argumentation-based negotiation, The Knowledge Engineering Review 18(4) (2003), 343-375. doi:10.1017/S0269888904000098.

[89] I. Rahwan and G.R. Simari (eds), Argumentation in Artificial Intelligence, Springer, Berlin, 2009.

[90] I. Rahwan, F. Zablith and C. Reed, Laying the foundations for a world wide argument web, Artificial Intelligence 171 (2007), 897-921. doi:10.1016/j.artint.2007.04.015.

[91] C. Reed and G.R. Araucaria, Software for argument analysis, diagramming and representation, International Journal of AI Tools 14(3-4) (2004), 961-980. doi:10.1142/S0218213004001922.

[92] C. Reed and F. Grasso, Recent advances in computational models of natural argument, International Journal of Intelligent Systems 22(1) (2007), 1-15. doi:10.1002/int.20187.

[93] C. Reed and T.J. Norman (eds), Argumentation Machines. New Frontiers in Argument and Computation, Kluwer Academic Publishers, Dordrecht, 2004.

[94] C. Reed, D. Walton and F. Macagno, Argument diagramming in logic, law and artificial intelligence, The Knowledge Engineering Review 22(1) (2007), 87-109. doi:10.1017/S0269888907001051.

[95] R. Reiter, A logic for default reasoning, Artificial Intelligence 13 (1980), 81-132. doi:10.1016/0004-3702(80)90014-4.

[96] E.L. Rissland and K.D. Ashley, A case-based system for trade secrets law, in: Proc. of the 1st International Conference on Artificial Intelligence and Law (ICAIL 1987), ACM Press, New York (New York), 1987, pp. 60-66.

[97] E.L. Rissland, K.D. Ashley and R.P. Loui, Ai and law: A fruitful synergy, Artificial Intelligence 150(1-2) (2003), 1-15. doi:10.1016/S0004-3702(03)00122-X.

[98] H. Rittel and M. Webber, Dilemmas in a general theory of planning, Policy Sciences 4 (1973), 155-169. doi:10.1007/ BF01405730.

[99] P. Saint-Dizier and M. Stede, Special issue: Foundations of the language of argumentation, Argument \& Computation 8(2) (2017), 91-93. doi:10.3233/AAC-170018.

[100] G.R. Simari and R.P. Loui, A mathematical treatment of defeasible reasoning and its applications, Artificial Intelligence 53 (1992), 125-157. doi:10.1016/0004-3702(92)90069-A.

[101] M. Stede and J. Schneider, Argumentation Mining, Morgan \& Claypool Publishers, 2018.

[102] C. Tempich, E. Simperl, M. Luczak, R. Studer and H. Pinto, Argumentation-based ontology engineering, IEEE Intelligent Systems 22(6) (2007), 52-59. doi:10.1109/MIS.2007.103.

[103] M. Thimm, A probabilistic semantics for abstract argumentation, in: Proc. of the 20th European Conference on Artificial Intelligence (ECAI 2012), IOS Press, Amsterdam, 2012, pp. 750-755.

[104] M. Thimm, S. Villata, F. Cerutti, N. Oren, H. Strass and M. Vallati, Summary report of the first international competition on computational models of argumentation, AI magazine 37(1) (2016), 102-102. doi:10.1609/aimag.v37i1.2640. 
[105] F. Toni, A generalised framework for dispute derivations in assumption-based argumentation, Artificial Intelligence 195 (2013), 1-43. doi:10.1016/j.artint.2012.09.010.

[106] F. Toni, A tutorial on assumption-based argumentation, Argument \& Computation 5(1) (2014), 89-117. doi:10.1080/ 19462166.2013.869878.

[107] S.E. Toulmin, The Uses of Argument, Cambridge University Press, Cambridge, 1958.

[108] L. van der Torre and S. Vesic, The principle-based approach to abstract argumentation semantics, in: Handbook of Formal Argumentation, P. Baroni, D. Gabbay, M. Giacomin and L. van der Torre, eds, College Publications, London, 2018, pp. 797-837.

[109] F.H. van Eemeren, B. Garssen, E.C.W. Krabbe, A.F. Snoeck Henkemans, B. Verheij and J.H.M. Wagemans, Handbook of Argumentation Theory, Springer, Berlin, 2014.

[110] F.H. van Eemeren and R. Grootendorst, Argumentation, Communication, and Fallacies. A Pragma-Dialectical Perspective, Lawrence Erlbaum, Hillsdale, 1992.

[111] T. van Gelder, Enhancing deliberation through computer supported argument visualization, in: Visualizing Argumentation: Software Tools for Collaborative and Educational Sense-Making, P.A. Kirschner, S.J.B. Shum and C.S. Carr, eds, Springer, 2003, pp. 97-115.

[112] B. Verheij, Two approaches to dialectical argumentation: Admissible sets and argumentation stages, in: Proc. of the 8th Dutch Conference on Artificial Intelligence (NAIC 96), J.J. Meyer and L.C. van der Gaag, eds, Universiteit, Utrecht, Utrecht, 1996, pp. 357-368.

[113] B. Verheij, DefLog: On the logical interpretation of prima facie justified assumptions, Journal of Logic and Computation 13(3) (2003), 319-346. doi:10.1093/logcom/13.3.319.

[114] B. Verheij, Dialectical argumentation with argumentation schemes: An approach to legal logic, Artificial Intelligence and Law 11(1-2) (2003), 167-195. doi:10.1023/B:ARTI.0000046008.49443.36.

[115] B. Verheij, Virtual Arguments. On the Design of Argument Assistants for Lawyers and Other Arguers, T.M.C. Asser Press, The Hague, 2005.

[116] G.A.W. Vreeswijk, Abstract argumentation systems, Artificial Intelligence 90 (1997), 225-279. doi:10.1016/S00043702(96)00041-0.

[117] D.N. Walton, Argumentation Schemes for Presumptive Reasoning, Lawrence Erlbaum, Mahwah, New Jersey, 2005.

[118] D.N. Walton and E.C.W. Krabbe, Commitment in Dialogue. Basic Concepts of Interpersonal Reasoning, State University of New York Press, Albany (New York), 1995.

[119] D.N. Walton, C. Reed and F. Macagno, Argumentation Schemes, Cambridge University Press, Cambridge, 2008. 